\title{
VORTEILE VON COMPLIANCE UND RISIKOMANAGEMENT
}

\section{ORIGINALER ARTIKEL}

SILVA, Lilian Reis da ${ }^{1}$

SILVA, Lilian Reis da. Vorteile von Compliance und Risikomanagement. Revista Científica Multidisciplinar Núcleo do Conhecimento. Jahr. 06, Hrsg. 12, Vol. 04, S. 123 147. Dezember 2021. ISSN: 2448-0959, Zugangslink: https://www.nucleodoconhecimento.com.br/business-administration-de/vorteile-voncompliance, DOI: 10.32749/nucleodoconhecimento.com.br/business-administrationde/vorteile-von-compliance

\section{ZUSAMMENFASSUNG}

Compliance ist ein Programm, das darauf abzielt, Unternehmen vor Finanzbetrug, Korruption, unangemessenem Verhalten und/oder Verhalten von mit innen verbundenen Mitarbeitern zu schützen, um zu verhindern, dass ihr guter Ruf und ihre Finanzkraft untergraben werden. Es ist ein Werkzeug, dessen Zweck es ist, Regeln, Standards und Richtlinien für interne Prozesse innerhalb von Organisationen festzulegen. Es wurde 1970 in den Vereinigten Staaten entwickelt und seine Praxis wurde durch die Verabschiedung des FCPA (Foreign Corrupt Practices Act) Pioneer Law in ein Rechtsinstitut umgewandelt, das durch den Watergate-Fall motiviert war, an dem der damalige Präsident Richard Nixon und Mitglieder beteiligt waren seiner Regierung durch die Zahlung von Bestechungsgeldern in der Forschung zugunsten seiner Wiederwahl. In diesem Zusammenhang ist die Leitfrage dieses Artikels: Wie wirken die Vorteile des Compliance- und Risikomanagement-Programms zusammen, um die Risiken von Betrug, illegalen Handlungen und Korruption innerhalb von Organisationen zu reduzieren? Ziel dieser Studie war es, die Vorteile der Einführung des Compliance- und Risikomanagement Programms in öffentlichen und privaten Unternehmen, in ihren internen Prozessen und in ihren Beziehungen zu ihrem

\footnotetext{
${ }^{1}$ Nachdiplom in Betriebswirtschaftslehre, Ökonom und Buchhaltungstechnik.

RC: 103919

Verfügbar in: https://www.nucleodoconhecimento.com.br/business-administrationde/vorteile-von-compliance
} 
Tätigkeitsbereich darzustellen und ihre Zusammenarbeit bei der Betrugsbekämpfung aufzuzeigen. Die bibliografische Recherche wurde als Methodik verwendet, um die Vorteile von Betrugspräventionsprogrammen zu diskutieren. Es wurde festgestellt, dass die Compliance- und Risikomanagement Programme zusammen mit neuen Governance-Lösungen für die Informationstechnologie (IT) wie Enterprise Governance, Risk Management and Compliance (EGRC) wirksame Vorteile beim Risikoschutz bieten, Betrug und Korruption eindämmen.

Stichworte: Betrug innerhalb von Organisationen, Prävention von Korruptionsrisiken, Compliance.

\section{EINLEITUNG}

Die Erörterung der Vorteile von Compliance und Risikomanagement impliziert die Präsentation von Konzepten darüber, was ein Compliance-Programm ist und welche Risiken Unternehmen eingehen, wenn sie diese Art von Programm nicht einführen, angesichts der verschiedenen Arten von Betrug, einschließlich Korruption, Geldwäsche, Manipulationen mit internen Informationen und / oder wenn Mitarbeiter Handlungen begehen, die das Image einer Organisation schädigen können. kann sogar finanzielle Verluste verursachen.

Laut Michelotti (2020, S. 6) bietet die Einführung eines Compliance- und Risikomanagement Programms Transparenz für Organisationen und zeigt die Existenz ethischen Verhaltens; Darüber hinaus arbeitet es mit den Praktiken der guten Corporate Governance zusammen, die die "Reihe von Prozessen, nach denen Unternehmen geführt werden", ist.

Laut Sobreira Filho, Leite und Martins (2019, S. 23), "ist das Compliance-Programm ein Unternehmen Instrument, dessen Methodik für seine Umsetzung auf Geschäftsethik und Integrität als größtem Wert basiert".

Der Begriff Compliance, geboren aus dem englischen Verb "to comply", wird definiert, zusammengefasst von Coelho und Santos Jr (2021, S. 8) als "erfüllen die

RC: 103919

Verfügbar in: https://www.nucleodoconhecimento.com.br/business-administrationde/vorteile-von-compliance 
Auferlegungen der Rechtsordnung oder der internen Ordnung des Unternehmens" (Greifen des Originals). Zu diesem Konzept fügt Gonsales (2016) hinzu, dass "wenn Compliance am Anfang bedeutete, "den Regeln zu folgen", aus den großen brasilianischen Untersuchungen auch "dem Antikorruptionsgesetz folgen".

Es ist bemerkenswert, dass Compliance für öffentliche oder private Unternehmen unterschiedlicher Größe und Segmente gilt, die sich in allen Ländern befinden, Organisationen, die das Verlangen haben, mit Ethik, Moral und bei der Förderung der menschlichen, sozialen, wirtschaftlichen und finanziellen Entwicklung zu handeln.

Dieser Artikel wurde als bibliografische Recherche entwickelt, für die die folgende Frage definiert wurde: Wie wirken die Vorteile des Compliance- und Risikomanagement-Programms zusammen, um die Risiken von Betrug, illegalen Handlungen und Korruption innerhalb von Organisationen zu reduzieren? Das allgemeine Ziel bestand wiederum darin, die Vorteile der Einführung des Complianceund Risikomanagement Programms in öffentlichen und privaten Unternehmen, in ihren internen Prozessen und in ihren Beziehungen zu ihrem operativen Segment darzustellen und ihre Zusammenarbeit zur Betrugsbekämpfung aufzuzeigen.

\section{COMPLIANCE}

\subsection{BEGRIFF}

Damit ein Unternehmen in seinem Tätigkeits Segment und vor der Gesellschaft als zuverlässig und inspirierend für Sicherheit gilt, ist es wichtig, dass es Werte hat, die auf Moral, Ethik und Gerechtigkeit basieren. Es sollte mit Transparenz handeln, was es ihm ermöglicht, seine Glaubwürdigkeit aufzubauen, und auch menschliche Talente anziehen, die seine Handlungsfähigkeit verbessern und auf sein Wachstum abzielen (JORGE und TOMAZ, 2018).

In dieser Hinsicht ist der Kampf gegen Betrug für eine Organisation, die mit Ethik, guten Prinzipien und Wettbewerbsfähigkeit handeln kann, ein grundlegendes Problem, das

RC: 103919

Verfügbar in: https://www.nucleodoconhecimento.com.br/business-administrationde/vorteile-von-compliance 
jede Art von Illegalität verhindert, durch die Annahme von Praktiken und Prozessen zum Schutz ihrer Werte und Ziele, die Annahme eines Compliance-Programms.

Compliance setzt "beitritt und Einhaltung von Normen und Vorschriften" voraus. Laut Antonik apud Jorge und Tomaz (2018, S. 108) kommt "compliance" aus dem Englischen, "to comply", was übersetzt "Regeln, Anweisungen, Normen, Richtlinien oder einfach auf einen Befehl reagieren"bedeutet.

Coelho und Santos Júnior (2021, S. 8) weisen darauf hin, dass "das Ziel der Compliance-Standards darin besteht, sich auf das zu erreichende Ergebnis zu konzentrieren, dh die Risiken zu vermeiden, die sich aus der Begehung von persönlichem oder organisatorischem Verhalten ergeben, das als illegal oder unvereinbar mit den Grundsätzen, Missionen, Visionen oder Zielen eines Unternehmens angesehen wird".

Im Laufe der Zeit, zwischen 1907 und 1977, sind in den Vereinigten Staaten Gesetze mit internationaler Reichweite entstanden, um Bürger, Unternehmen und Regierungsbehörden, die sich an Korruption Handlungen beteiligen, zu kontrollieren und zu bestrafen.

Laut Nascimento (2018) wurde Compliance in den Vereinigten Staaten um die 1970er Jahre herum geschaffen und als Rechtsinstitut aus der Verabschiedung des Pioneer Law zur Bekämpfung von Korruption oder - Foreign Corrupt Practices Act (FCPA) erhoben. Eine solche Gesetzgebung wurde nach dem Watergate-Fall entwickelt und umgesetzt, der im Rücktritt des damaligen Präsidenten der Vereinigten Staaten, Richard Nixon, gipfelte.

Furtado und Rocha (2015, apud MICHELOTTI, 2020, S. 7-8) berichten, dass in der Zeit vor dem FCPA-Gesetz die Praktiken des Kapitalismus bei der Öffnung neuer Märkte die Zahlung von Bestechungsgeldern beinhalteten, "die als notwendiger und natürlicher Schritt im Unternehmensumfeld angesehen wurden. Es bestand die deutlichste Möglichkeit, dass das Bestechungsgeld als Aufwand in der Bilanz verbucht

RC: 103919

Verfügbar in: https://www.nucleodoconhecimento.com.br/business-administrationde/vorteile-von-compliance 
wurde. Und Bestechung, um Verträge oder Konzessionen in diesen Ländern zu erhalten, war Teil der Spielregeln."

Da Korruption ein globales Problem ist und von Anfang an Regierungen, Menschen, öffentliche und/oder private Institutionen betrifft, stellt Ubirajara Costódio Filho, auf die sich Nascimento (2021) bezieht, fest, dass es sich um etwas handelt, das allen sozialen Bereichen schadet, in Bezug auf die Umwelt, den freien Wettbewerb, die öffentlichen Finanzen, den internationalen Handel, Demokratie und republikanische Gebote.

Immer noch über Korruption, Nascimento (2021, n.p.) berichtet auch Eduardo Cambi, um hervorzuheben, dass:

a corrupção atrasa o desenvolvimento econômico e social. Restringe a vontade soberana do povo. Apropria a coisa pública para a realização de interesses privados. Gera promiscuidade entre o poder público e o poder econômico. Concentra renda. Ressalta privilégios e desigualdades. Impede a universalização de políticas públicas e a concretização de direitos fundamentais. Destrói a cidadania e enfraquece a democracia.

Der Zweck des Foreign Corrupt Practices Act (FCPA) war es, betrügerische Handlungen von Unternehmen zu bestrafen, die Bestechungsgelder für den öffentlichen Dienst anbieten / zahlen würden, mit dem Ziel, Unternehmen auf der ganzen Welt zu expandieren. Seit der Annahme der im FCPA-Gesetz vorgeschriebenen Praktiken waren alle Organisationen verpflichtet, interne und externe Vorschriften zu befolgen, die auf den ethischen und moralischen Fragen basieren, die für die kommerziellen und wirtschaftlichen Beziehungen zu ihren Partnern erforderlich sind (NASCIMENTO, 2018).

\subsection{ENTSTEHUNG VON RECHTSVORSCHRIFTEN FÜR COMPLIANCE-PRAKTIKEN}

Laut Nascimento (2018, S. 26) begann die globale Bewegung zur Bekämpfung von Bestechung und der tief verwurzelten Praxis der Korruption "mit dem Foreign Corrupt Practices Act (FCPA) oder dem amerikanischen Gesetz gegen korrupte Praktiken".

RC: 103919

Verfügbar in: https://www.nucleodoconhecimento.com.br/business-administrationde/vorteile-von-compliance 
Dieser Autor stellt klar, dass der "Grad der Korruption in einem bestimmten Land je nachdem, wie multinationale Unternehmen dort handeln variiert".

Unter den verifizierten Korruptionsraten gibt Nascimento (2018, S. 26-27) den Anteil der Unternehmen auf der ganzen Welt an, in denen die illegale Korruption und Geldwäsche fortbesteht:

$23 \%$ das empresas na União Europeia e Europa Ocidental; $66 \%$ das empresas na América Latina; $64 \%$ das empresas na Ásia; $95 \%$ das empresas na Europa Ocidental e da Ásia Central; $84 \%$ das empresas do Oriente Médio e do Norte da África; $90 \%$ das empresas da África Subsaariana.

Dieser Autor (NASCIMENTO, 2018, S. 26-27) sagt, dass die wirtschaftliche Globalisierung "das globale Verbrechen der Korruption im Zusammenhang mit Geldwäsche und Offshore-Korruption hervorgebracht hat".

Es wird daher festgestellt, dass die Literatur zu Compliance- und Risikomanagement Programmen zeigt, dass zu einem bestimmten Zeitpunkt mehrere Länder und ihre Regierungen beschlossen haben, illegalen Praktiken - sowohl in öffentlichen Einrichtungen als auch in privaten Organisationen - entgegenzutreten, dass sie fortan gezwungen wurden Straf- und restriktive Maßnahmen für Bürger und Länder zu ergreifen, die unter anderem Betrug, Korruption und Geldwäsche praktizierten, was zu internationalen Verträgen führte.

Länder, die Betrugsprävention Programme einführen, sind Unterzeichner der Organisation für wirtschaftliche Zusammenarbeit und Entwicklung (OECD), darunter: Deutschland, Australien, Österreich, Belgien, Kanada, Chile, Korea, Dänemark, Slowenien, Spanien, Vereinigte Staaten, Estland, Finnland, Frankreich, Griechenland, Ungarn, Irland, Island, Israel, Italien, Japan, Lettland, Luxemburg, Mexiko, Norwegen, Neuseeland, Niederlande, Peru, Polen, Portugal und Vereinigtes Königreich (NASCIMENTO, 2021, s.p.).

Um zu klären, wie diese Länder sich für die Einführung guter Corporate-GovernancePraktiken entschieden haben, ist es wichtig, die Entstehung von US-Institutionen und 
-Gesetzen herbeizuführen, die die Einführung von Schutzinstituten in Bezug auf die verschiedenen Arten von illegalen Gegenorganisationen ermöglichten, was zur Verabschiedung von Gesetzen für die gleichen Zwecke auf der ganzen Welt führte.

1907 fand die Haager Konferenz statt, die "ein internationaler Gerichtshof zur Untersuchung internationaler Konflikte" ist. Damit wurde ein Spielraum für die Stiftung entwickelt der Bank für Internationalen Zahlungsausgleich (BIZ) (Bank for International Settlements - BIS), um "die Zusammenarbeit zwischen Zentralbanken zu mehr Finanzstabilität" zu fördern. Dieses Ziel wird von Experten als "die ersten Wurzeln der Compliance" angesehen (NASCIMENTO, 2021, s.p.).

Im April 1948 wurde in Portugal die Europäische Organisation für wirtschaftliche Zusammenarbeit (OEEC) gegründet, die auf die wirtschaftliche Zusammenarbeit zwischen den europäischen Ländern abzielte. Seine ursprüngliche Zusammensetzung hatte 18 Länder, um hervorzuheben: Österreich, Belgien, Dänemark, Frankreich, Griechenland, Holland, Irland, Island, Italien, Luxemburg, Norwegen, Portugal, Vereinigtes Königreich, Schweden, Schweiz, Türkei und Westdeutschland.

Ebenfalls 1948 wurde die Organisation Amerikanischer Staaten (OAS) (Organization of American States - OAS), die auf vier Säulen beruhte: "Demokratie, Menschenrechte, Sicherheit und Entwicklung". (NASCIMENTO, 2021, s.p.).

Dennoch hebt Nascimento (2021, s.p.) hervor, was Sanches und Renee (2017, S. 18) über die OAS verteidigen: "Diese Konvention zielt darauf $a b$, die notwendigen Mechanismen zu fördern und zu stärken, um Korruption bei der Ausübung öffentlicher Funktionen sowie Korruption Handlungen, die speziell mit ihrer Ausübung verbunden sind, zu verhindern, aufzudecken und zu bestrafen".

Im Dezember 1960 unterzeichneten die OEEC-Mitglieder mit den USA und Kanada ein neues Übereinkommen, das die Organisation für wirtschaftliche Zusammenarbeit und Entwicklung (OECD) schuf und dann die OEEC ersetzte. So entstand 1961 die Organisation für wirtschaftliche Zusammenarbeit und Entwicklung (OECD) (Organisation for Economic Co-operation and Development (OECD), als Nachfolgerin

RC: 103919

Verfügbar in: https://www.nucleodoconhecimento.com.br/business-administrationde/vorteile-von-compliance 
der Organisation für Europäische wirtschaftliche Zusammenarbeit, deren Ziele es waren, "die wirtschaftliche Entwicklung und finanzielle Stabilität der Mitgliedsländer abzudecken" (NASCIMENTO, 2021, s.p.).

1975 wurde der Basler Ausschuss für Bankenaufsicht geschaffen (BCBS) (Basel Committee on Banking Supervision - BCBS), dessen Zweck es war, "beste Finanz Praktiken zu regulieren und zu beaufsichtigen". 1998 förderte sie eine Standardisierung der für Finanzinstitute geltenden Vorschriften mit dem Ziel, gute Corporate-Governance-Praktiken aufrechtzuerhalten (NASCIMENTO, 2021, s.p.).

Im Jahr 1977 wurde angesichts des Watergate-Skandals das amerikanische Gesetz gegen die Praktiken der Korruption im Ausland (FCPA) entwickelt, das 1. Antikorruptionsgesetz, das später in den Jahren 1988 und 1998 erlassen wurde. Laut Nascimento (2021, s.p.) mit dem Ziel, "zivil-, strafrechtliche und administrative Strafen gegen natürliche und juristische Personen gegen korrupte Praktiken zu verhängen, den Foreign Corrupt Practices Act (FCPA) oder das amerikanische Gesetz gegen korrupte Praktiken" (NASCIMENTO, 2021, s.p.).

Es ist bemerkenswert, dass das FCPA als "Verbrechen die Zahlung von Bestechungsgeldern direkt oder indirekt, Zahlungen an ausländische Beamte, politische Parteien, die Zahlung nicht prägnant aufgezeichnet, es müssen Bücher der Buchhaltungsunterlagen vorhanden sein" (NASCIMENTO, 2021, s.p.).

Solche Untersuchungen wurden von der Getúlio Vargas Stiftung (FGV) (2021, s.p.) durchgeführt, die über die Entstehung der Securities and Exchange Commission (SEC) berichtete, die "eine Verwaltungsorganisation zur Überwachung und Bekämpfung von Korruptionspraktiken bei internationalen Transaktionen" ist.

Die SEC "fand Praktiken illegaler Zahlungen an Beamte von durchschnittlich 400 privaten und öffentlichen Unternehmen in den USA sowie ausländischen Unternehmen". In einer solchen Erklärung stellte die SEC fest, dass "sie zusammen mehr als 300 Millionen Dollar an Bestechungsgeldern an ausländische Regierungsbeamte gezahlt haben" (FGV, 2021, s.p.).

RC: 103919

Verfügbar in: https://www.nucleodoconhecimento.com.br/business-administrationde/vorteile-von-compliance 
Im Jahr 1989 wurde - für gemeinsame Maßnahmen mit der OECD - die Internationale Finanzielle Aktionsgruppe (FATF) (Financial Action Task Force - FATF) gegründet, die auf der Grundlage internationaler Gesetze politische Entscheidungen sowie Gesetzes- und Regulierungsreformen zur Bekämpfung von Geldwäsche und Terrorismusfinanzierung durchführen soll. Die FATF ist auch bei der Zusammensetzung der periodischen Evaluierung Mechanismen anderer Länder tätig (NASCIMENTO, 2021, s.p.).

In der Praxis brachte der OAS-Text die 1996 unterzeichnete Interamerikanische Konvention gegen Korruption ein, die darauf abzielte, "die Interessen der Vereinigten Staaten in Bezug auf Terrorismus und Korruption zu schützen".

Im Jahr 2002 wurde der Sarbanes Oxley Gesetz (SOX) (Sarbanes-Oxley Act SARBOX) eingeführt, um eine bessere "Unternehmensführung, Überwachung und Prävention schädlicher Praktiken, Erhöhung der Kontrollen und Transparenz" zu fördern. Mit der Schaffung des Arbeitsaufsicht Gremiums unabhängiger Wirtschaftsprüfer führte es auch unabhängige Prüfungen für Verdachtsfälle ein, stärkte die "Verantwortung für Unternehmens- oder kriminellen Betrug, erhöhte die Offenlegung von Finanzinformationen, erhöhte die Strafen für Wirtschaftskriminalität (NASCIMENTO, 2021, sp).

Im Jahr 2010 erscheint im Vereinigten Königreich der (UK Bribery Act (BA), der vier Straftaten beschreibt: "aktive Korruption öffentlicher und privater Subjekte, passive Korruption öffentlicher und privater Subjekte, Korruption ausländischer öffentlicher Agenten, Unternehmensversagen bei der Korruptionsprävention" ( NASCIMENTO, 2021, sp).

Im Gegenzug entwickelten Madruga und Belloto einen Leitfaden, der 10 Säulen vorstellt, die nach ihrer Annahme die Umsetzung eines effizienten ComplianceProgramms ermöglichen (NASCIMENTO, 2018, S. 8-9):

1. Compromisso de todos os níveis da gerência e política anticorrupção claramente articulada; 2. Código de conduta e política e procedimentos de

RC: 103919

Verfügbar in: https://www.nucleodoconhecimento.com.br/business-administrationde/vorteile-von-compliance 
compliance; 3. Fiscalização interna do programa, autonomia da área de compliance e recursos adequados; 4. Avaliação dos riscos da empresa; 5. Treinamentos e aconselhamento contínuo; 6. Incentivos e medidas disciplinares; 7. Investigações (due diligence) de terceiros e de pagamentos; 8. Sistema interno de denúncias (possibilitando denúncias confidenciais) e sistema de investigações internas; 9. Melhoria contínua: testes e revisões periódicas do programa de compliance; 10. Fusões e Aquisições: Investigações (due diligence) pré-fusões e aquisições e integrações pós-aquisições e fusões.

In Bezug auf Brasilien und trotz des Erlasses des Gesetzes Nr. 12.846/13, dessen Zweck die Annahme rechtlicher Maßnahmen und interner Verfahren ist, die von Organisationen durch die Annahme eines Verhaltenskodex zur Bekämpfung der Korruption einzuhalten sind, wurde der Beitritt zur OECD von diesem Gremium angesichts der ständigen Fälle von Korruption noch nicht offiziell gemacht. Betrug und Geldwäsche, die hier auftreten (DURÃES und RIBEIRO, 2020).

\subsection{DIE BETRÜGERISCHEN PRAKTIKEN UND VERLUSTE, DIE VON DRITTEN GEGENÜBER ORGANISATIONEN VERURSACHT WERDEN}

Wenn Menschen, die mit einer bestimmten Organisation verbunden sind, Verhaltensweisen annehmen, die dem Image des Unternehmens schaden, haben ihre falschen Handlungen negative Auswirkungen auf das Unternehmen sowie auf die gesamte Gesellschaft. Es gibt viele Fälle von verwerflichen Verhalten, die nicht nur das Image erschüttern, sondern auch Verluste, Bußgelder und manchmal auch Konkurs mit sich bringen.

In Bezug auf die verschiedenen Arten von Betrug innerhalb von Regierungsorganisationen und -institutionen, wie das Durchsickern vertraulicher Informationen, passive / aktive Korruption, Geldwäsche, unter anderem, gibt Gonsales (2016) an, dass alle Studien im Zusammenhang mit solchem Betrug argumentieren, dass sie in jedem Unternehmen auftreten. Je mehr Ihre Führungskraft glaubt, dass nichts Falsches passiert, desto anfälliger wird Ihr Unternehmen.

In Brasilien gibt es betrügerische Praktiken, die in der gesamten Gesellschaft bekannt sind, wie z.B. "Fälschung der Lizenz eines Studenten oder Diebstahl von

RC: 103919

Verfügbar in: https://www.nucleodoconhecimento.com.br/business-administrationde/vorteile-von-compliance 
Kabelfernsehleitungen", auf die sich Condé et al. beziehen. (2015, S. 95); es sind Handlungen, die darin bestehen, die Bösgläubigkeit einiger und ihre klare Absicht, andere zu täuschen, zu demonstrieren. Leider sind es Handlungen, die von vielen Menschen praktiziert werden, aber die im Laufe der Zeit so üblich geworden sind, dass sie in der brasilianischen Kultur akzeptiert werden. Es sind Handlungen, die eine unethische und illegale Mentalität und ein unethisches Verhalten zeigen; Sie werden jedoch als nicht schädlich für die Gesellschaft angesehen, was unwahr ist.

Im Vergleich zu den oben genannten Aktionen haben Condé et al. (2015, S. 95) legt nahe, dass, wenn unethische, betrügerische und korrupte Praktiken von hochrangigen Führungskräften einer Organisation durchgeführt würden, sie von der Gesellschaft als schädlich und inakzeptabel angesehen würden.

In diesem Zusammenhang berichtet Cossenzo (2015, S. 35) Oliveira (2012, S. 115), um zu definieren, dass "ein Finanzbetrug auf der Grundlage der freiwilligen Handlungen seines Agenten mit der klaren Absicht durchgeführt wird, anderen finanziell zu schaden". Dieser Autor erklärt weiter, dass Betrug (i) intern sein kann wenn er von Mitarbeitern gegen die Organisation begangen wird; (ii) extern - wenn sie von Dritten begangen werden, die nicht Teil des Unternehmens sind; (iii) gemischt: wenn die betrügerischen Handlungen von internen und externen Agenten durchgeführt werden.

Nach dieser Erklärung lohnt es sich, ein Konzept im Zusammenhang mit Finanzbetrug hervorzuheben, das als "dem Geschäft innewohnendes" dieser Art von Institut angesehen wird und sich selbst als operationelle Risiken klassifiziert, die sowohl mit diesem Segment als auch mit "Menschen, Prozessen und Technologien" zusammenhängen (COSSENZO, 2015, S. 35).

Es sollte klargestellt werden, dass Compliance alle Arten von Betrug abdeckt, die in verschiedenen Geschäftssegmenten auftreten können, dh Compliance zielt darauf ab, die Risiken zu mindern, die durch persönliches oder organisatorisches Verhalten verursacht werden können (unabhängig davon, ob sie sich im Finanzsegment befinden 
oder nicht), wenn sie nicht in Übereinstimmung mit ethischem Verhalten und in Übereinstimmung mit den Richtlinien des Unternehmens handeln.

\subsection{EREIGNISSE, DIE DEM RUF VON US-UNTERNEHMEN SCHADEN}

Laut Haussen (2021, S. 9) "kann der Ruf einer Organisation durch unzusammenhängendes Verhalten, das sich aus der mangelnden Umsetzung eines Compliance-Programms ergibt, stark erschüttert werden", wie die Korruptionsfälle in den Vereinigten Staaten zeigen, die im Laufe der Jahre in den Medien gestritten sind, einschließlich des Watergate-Falls - der zur Verabschiedung des FCPA-Gesetzes führte. zusätzlich zu anderen, die in diesem Artikel erwähnt werden.

Unter den zahlreichen Fällen von Handlungen, die dem Image großer USUnternehmen schaden und ihre Glaubwürdigkeit gefährden, werden hier einige hervorgehoben, die in den Medien viel Aufmerksamkeit erregt haben, wie einige Berichte in elektronischen Medien zeigen. Dies sind Geschichten, die berichten, wie die mangelnde Implementierung eines Compliance-Programms zur Steuerung von Prozessen und Menschen innerhalb einer Organisation dazu führt, dass einige Mitarbeiter das Image und den Ruf weltweit bekannter Unternehmen mit verwerflichen Maßnahmen beschädigen.

Der erste Bericht von Vaz (2012), der von Zeitschrift Exame veröffentlicht wurde, bringt einen Fall, der im Riesen Apple aufgetreten ist, dessen Volltext hier vorgestellt wird.

A Empresa Apple foi acusada pelo Senado nos Estados Unidos de usar filiais no exterior para evitar o pagamento de bilhões de dólares em impostos. "A Apple não apenas transferiu seus lucros para um paraíso fiscal estrangeiro, mas criou um 'Santo Graal' de evasão fiscal ao montar entidades no exterior para driblar o fisco", disse o senador democrata Carl Levin.

Uma reportagem do new York Times revelou como a Apple tem feito para driblar bilhões de dólares em impostos nas suas operações nos Estados e em outros países. Segundo Martin A. Sullivan, um ex-economista do Tesouro americano, sem as táticas utilizadas para a gestão dos impostos, a empresa liderada por Tim Cook teria deixado pelo menos mais 2,4 bilhões de dólares em taxas federais nos EUA no ano passado. A empresa declarou ter pagado 3,3 bilhões de dólares em impostos ao redor do mundo em 2011 sobre os lucros

RC: 103919

Verfügbar in: https://www.nucleodoconhecimento.com.br/business-administrationde/vorteile-von-compliance 
de 34,2 bilhões de dólares, uma taxa de 9,8\%. A companhia declara que 30\% dos lucros vem dos Estados, caso o percentual pulasse para $50 \%$, os pagamentos saltariam em 2,4 bilhões de dólares. "É razoável esperar que $70 \%$ dos lucros viriam dos Estados Unidos”, calcula.

Abbildung 1: Apple-Symbol

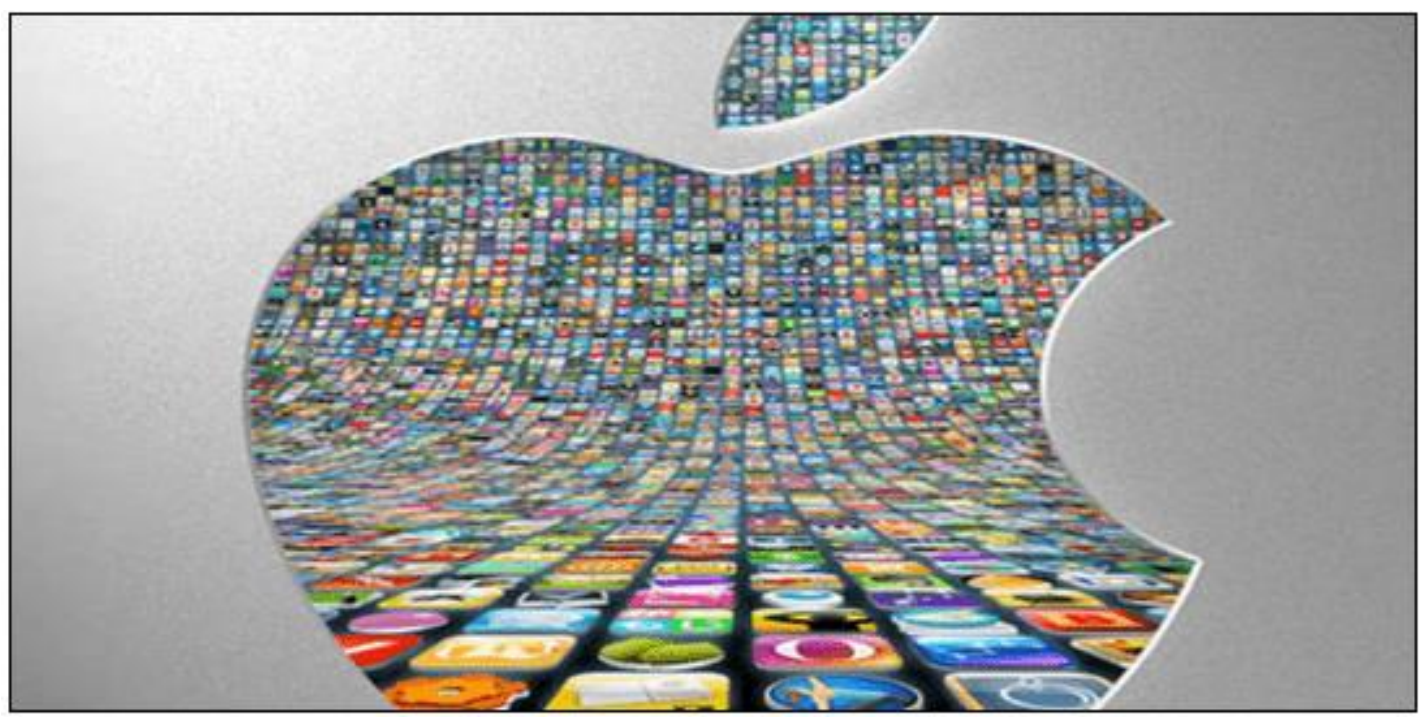

Quelle: Vaz (2012)

In Bezug auf mögliche Ursachen, die die Glaubwürdigkeit von Organisationen beeinträchtigen können, Souza; Maciel-Lima und Lupi (2018, S. 13) beziehen sich auf Gonsales (2016), um zu erklären, dass:

o valor de uma empresa está ligado à sua reputação e no caso de uma empresa ser citada em jornais e meios de comunicação, ainda que seja um ato ilícito isolado, acometido por um funcionário, a empresa sofrerá abalo a reputação e passa a ser conhecida no mercado como uma empresa corrupta.

Der zweite Bericht bezieht sich auf zwei verschiedene Situationen, die in zwei verschiedenen Einheiten in nordamerikanischen Restaurants des Taco BellNetzwerk auftraten, Fast Food inspiriert von der mexikanischen Küche, die dem McDonalds-Modell folgt.

Der erste ist in Melos Bericht (2014), der vom Zeitschrift Exame veröffentlicht wurde, dessen Text unten folgt.

RC: 103919

Verfügbar in: https://www.nucleodoconhecimento.com.br/business-administrationde/vorteile-von-compliance 
Publicar uma foto de si mesmo fazendo xixi em um prato do Taco Bell, nos Estados Unidos, não custou ao atendente Cameron Jankowski apenas o emprego. Indignados, hackers divulgaram os dados pessoais do "brincalhão" na internet, segundo o Huffington Post. Em 2012, Jankowski postou imagem no Twitter, mas disse que não chegou a servir a comida no restaurante. Ele chegou a deletar sua conta, mas acabou sem seu emprego na rede.

Abbildung 2: Elektronisches Bedienfeld von Taco Bell

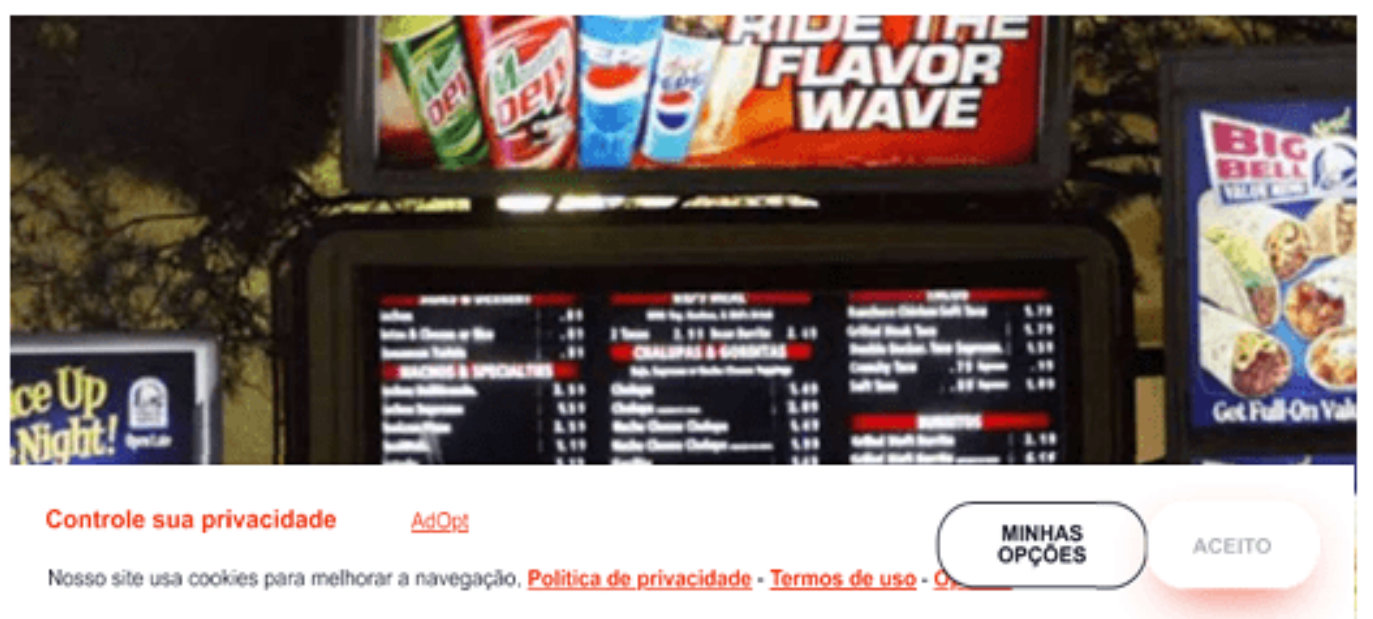

Quelle: Melo (2014)

Im Jahr 2013 hätte das gleiche Netzwerk einen weiteren Moment durchgemacht, der seiner Glaubwürdigkeit Schaden zugefügt hätte. Der Bericht stammt aus dem Zeitschrift Veja On Line (2014), dessen Text vollständig folgt.

Uma imagem de um funcionário da rede de comida mexicana Taco Bell lambendo uma pilha de tortilhas foi postada na página da empresa no Facebook. A foto, de autoria do usuário Jj O'Brien Nolan, é seguida da legenda "isto com certeza diz muito sobre seus empregados, o tratamento da comida e o que eles postam na internet.

Apesar de inicialmente acreditar que a foto fosse uma brincadeira, a empresa demitiu o empregado. Além disso, em resposta, a Taco Bell divulgou um comunicado oficial, além de comentar a postagem de Nolan no Facebook. A empresa se defendeu afirmando que todas as unidades têm rígidos procedimentos de manuseamento e tolerância zero para violação dos alimentos.

RC: 103919

Verfügbar in: https://www.nucleodoconhecimento.com.br/business-administrationde/vorteile-von-compliance 
Abbildung 3: Taco Bell-Mitarbeiter leckt einen Haufen Tortillas

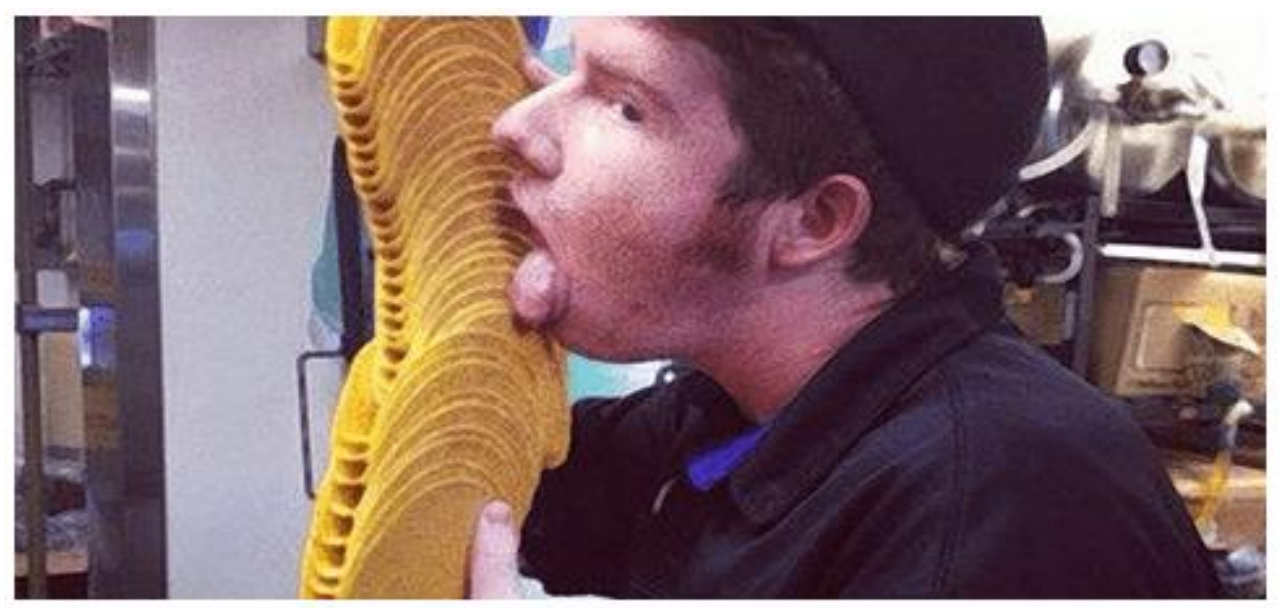

Quelle: Veja Online (2014)

Ein dritter zu erwähnender Fall wurde in einem Bericht von Melo (2014, veröffentlicht vom ZeitschriftExame) veröffentlicht, der sich in einem der Restaurants des KFC Network, Nordamerika, ereignete, dessen Originaltext hier wiedergegeben ist.

Dies war der Fall, als eine ehemalige Mitarbeiterin der KFC-Restaurantkette in den Vereinigten Staaten ein Foto auf Facebook postete, als sie buchstäblich das Kartoffelpüree leckte. Sie und ihr befreundeter Fotograf wurden gefeuert. Das Netzwerk musste sich damit rechtfertigen, dass das Essen nicht serviert wurde. 
Abbildung 4 - KFC-Mitarbeiter leckt Kartoffelpüree
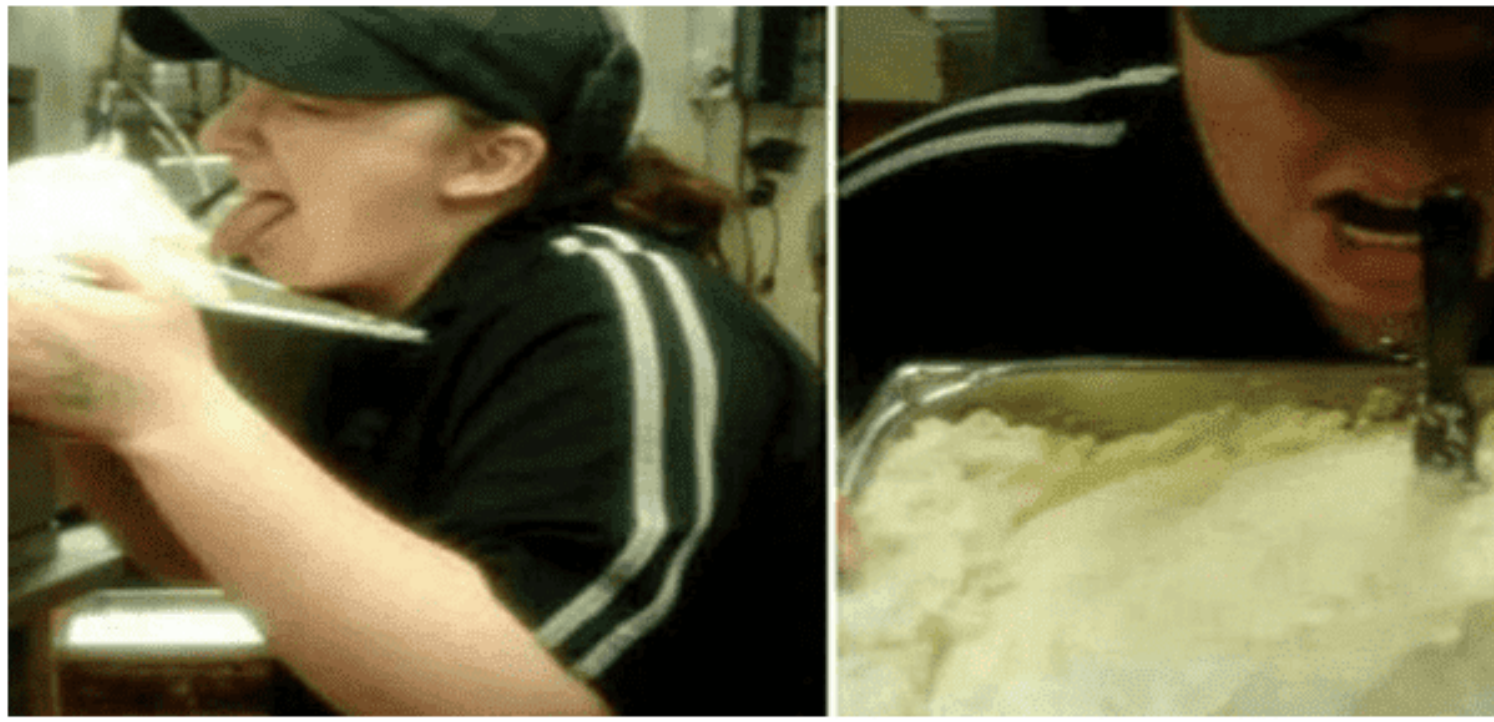

Quelle: Melo (2014)

Die dargestellten Fälle von unethischem Verhalten spiegeln die Bedeutung der Einführung von Compliance- und Risikomanagement-Praktiken wider. Aus Sicht von Coelho und Santos Júnior (2021, S. 26) "reicht es nicht aus, dass das Unternehmen die finanziellen Voraussetzungen hat, in Werbung und Marketing zu investieren, um marktführend zu sein."

In der Praxis wird bestätigt, dass Unternehmen ständig auf dem Laufenden bleiben und auf neue Marketing Praktiken achten müssen, zusätzlich zu Strategien, die es ihnen ermöglichen, in ihrem Tätigkeits Segment wettbewerbsfähig zu sein, bessere Dienstleistungen und wettbewerbsfähige Preise anzubieten und ihren Ziel Verbrauchern Wahlmöglichkeiten zu bieten.

In diesem Sinne argumentieren Coelho und Santos Júnior (2021, S. 27), dass "zu den Faktoren, die das Niveau der Wettbewerbsfähigkeit der Unternehmen beeinflussen, gehören: Innovationskapazität, Qualitätsprodukte und -dienstleistungen, kommerzielle Unterschiede, Produktions- und Lagerkapazität, geografische Reichweite, Marketingmaßnahmen; unter anderem."

RC: 103919

Verfügbar in: https://www.nucleodoconhecimento.com.br/business-administrationde/vorteile-von-compliance 


\section{DIE RISIKEN DER COMPLIANCE}

Durch das Versäumnis, Betrugspräventions- und -minderungsprogramme in ihren organisatorischen Prozessen zu übernehmen, sind Unternehmen dem Ereignis verschiedener illegaler Ereignisse ausgesetzt, da "Betrug in allen Unternehmen auftritt". Darüber hinaus sind sie anfällig für zwei Arten von sehr schädlichen Risiken: die Erschütterung ihres Rufs und die strafen, die im neuen Antikorruptionsgesetz vorgesehen sind (GONSALES, 2016).

Der Begriff "Risiken der Compliance" bezieht sich auf die Risiken, denen Unternehmen durch regulatorische Sanktionen, finanzielle Verluste und/oder Verluste ihres Images, Risiken, die sich aus der Nichteinhaltung von Vorschriften, Verhaltenskodizes und gutem Verhalten ergeben, ausgesetzt sind (COELHO und SANTOS JR, 2021, S. 8).

Laut Gonsales (2016) haben 43\% der brasilianischen Unternehmen noch keine Compliance-Programme und -Richtlinien für ihre Prozesse und ihren Schutz eingeführt, aber bald werden alle dazu gezwungen sein, um ihren Ruf zu schützen, da "der Wert eines Unternehmens mit seinem Ruf zusammenhängt.

Es ist eine Realität, die das nationale kommerzielle und industrielle Umfeld verändert hat, die sich aus den Untersuchungen von Mensalão und der Operação Lava Jato und danach ergibt, da die Demontage von Finanzbetrug und nachgewiesener Korruption und Geldwäsche die Notwendigkeit eines neuen Geschäftsumfelds zeigt, das Transparenz und Integrität in der Geschäftstätigkeit erfordert. Die Bestrafungen und Verhaftungen von hochrangigen Führungskräften und hochrangigen Regierungsbeamten erfolgten vor dem Hintergrund des neuen Antikorruptionsgesetzes, das im Land eingeführt wurde (GONSALES, 2016).

Von PwC Brasil (2020, S. 4) recherchierte Daten belegen, dass das Compliance-Risiko in Bezug auf Betrug und Wirtschaftskriminalität am häufigsten bei Organisationen auf nationaler und internationaler Ebene auftritt. Diese Untersuchung ergab, dass sie in Brasilien zu den drei häufigsten Betrugsarten gehören: (1) Bestechung und Korruption, 
(2) Buchführungs Betrug und (3) Betrug durch den Verbraucher. Seit Durchführung der Umfrage im Jahr 2020 hat sich gezeigt, dass in den letzten zwei Jahren (zwischen 2018 und 2019) die häufigsten Betrugsfälle, die in den Spitzenpositionen auftraten, waren: (a) Vermögens Diebstahl) Betrug beim Einkauf und (c) Bestechung und Korruption.

Laut PwC (2020, S. 4) waren Vergleichsdaten des Rankings der Betrügereien, die weltweit an der Spitze stehen, (1) Betrug durch Verbraucher, (2) Cyberkriminalität und (3) Diebstahl von Vermögenswerten. Da die Umfrage im Jahr 2020 durchgeführt wurde, wurde gezeigt, dass in den letzten beiden Jahren (zwischen 2018 und 2019) die die häufigsten Betrügereien, die in den Spitzenpositionen auftauchen, waren, waren: (a) Betrug durch den Verbraucher, (b) Buchhaltung Betrug, (c) unlauterer Wettbewerb, (d) Personalberatung und (e) Bestechung und Korruption.

Abbildung 5 zeigt die Daten in der internen und externen Perspektive. 
Abbildung 5 - Die häufigsten Arten von Straftaten

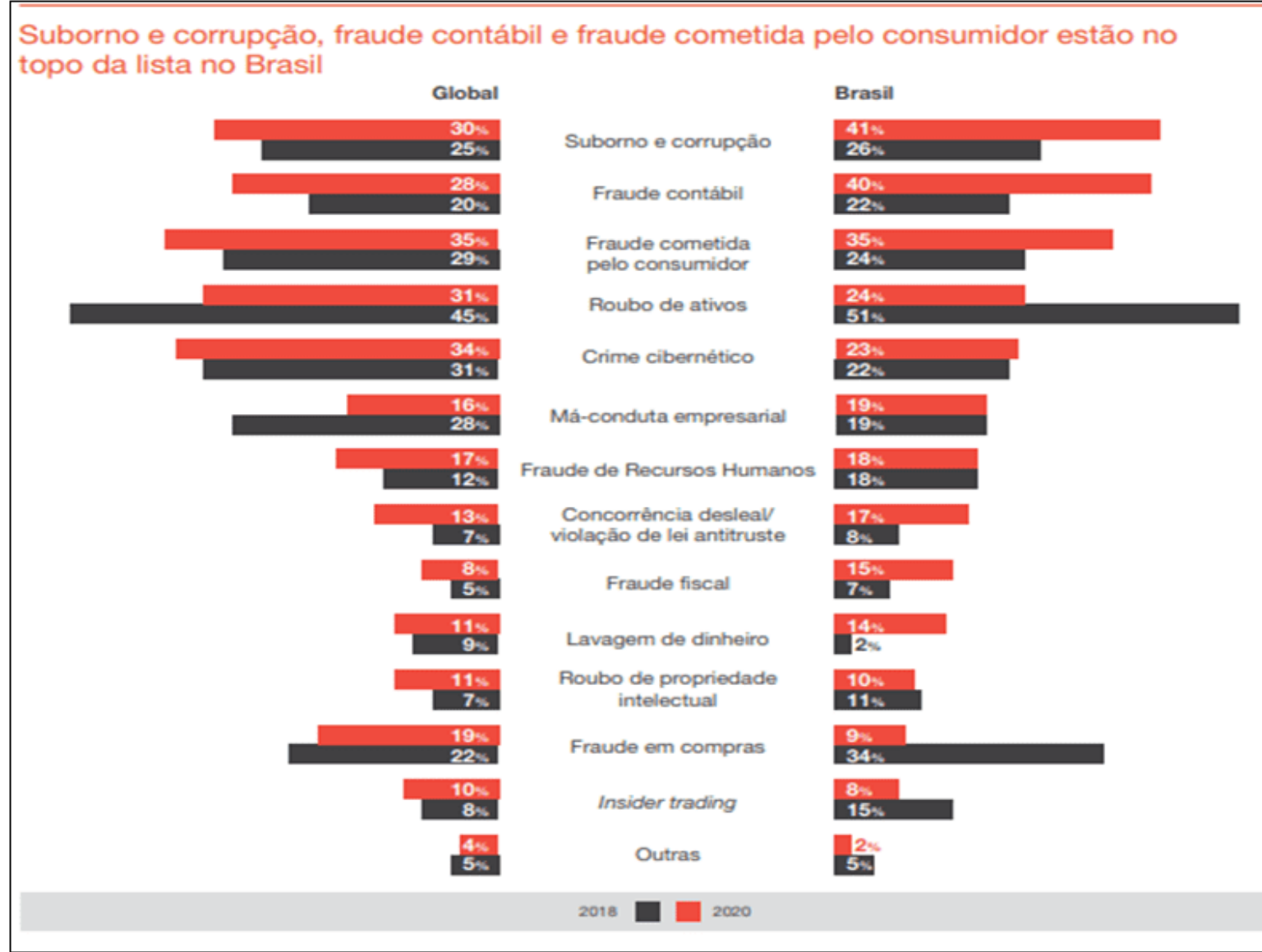

Quelle: PwC Brasil (2020, S. 5)

Dabei handelt es sich um Wirtschaftskriminalität, die nicht nur dem Vermögen der Organisationen schadet, sondern auch deren Ruf und Image beeinträchtigt und sogar bankrott geht. Aus dieser Perspektive ermöglichen Compliance-Programme die Vermeidung von Risiken, illegalen Handlungen und unethischem Verhalten, indem ein spezifisches Programm implementiert wird, das eindeutig den Ethikkodex und die interne Richtlinie der Organisation zu befolgen enthält und „Wachstum, Investitionen, Glaubwürdigkeit, Sicherheit und Schutz" (PwC, 2020).

In diesem Sinne stellt Oliveira (2012 apud COSSENZO, 2015, S. 35) fest, dass die Präventionspflege im Wesentlichen aus der Verpflichtung bestehen sollte, die Unternehmen intern in Bezug auf ethisches Verhalten für Entscheidungen und

RC: 103919

Verfügbar in: https://www.nucleodoconhecimento.com.br/business-administrationde/vorteile-von-compliance 
Verhaltensweisen, die ihre Geschäfts- und Betriebsprozesse durchdringen, etablieren müssen und sogar präventive Praktiken anwenden können, die sich auf die Geschichte von Mitarbeitern, Lieferanten und Kunden beziehen, um Stärkung einer internen und externen Betrugsbekämpfungskultur.

Unter den Risiken der Compliance ist die Theorie des Betrugsdreiecks zu erwähnen, die drei Aspekte hervorhebt, die die Wahrscheinlichkeit illegaler Handlungen gegen Organisationen erhöhen: Druck, Chancen und Rationalisierung, wie von Condé, Almeida und Quintal (2015) erläutert. Dieses Dreieck kann in Abbildung 6 betrachtet werden.

Abbildung 6: Betrugsdreieck

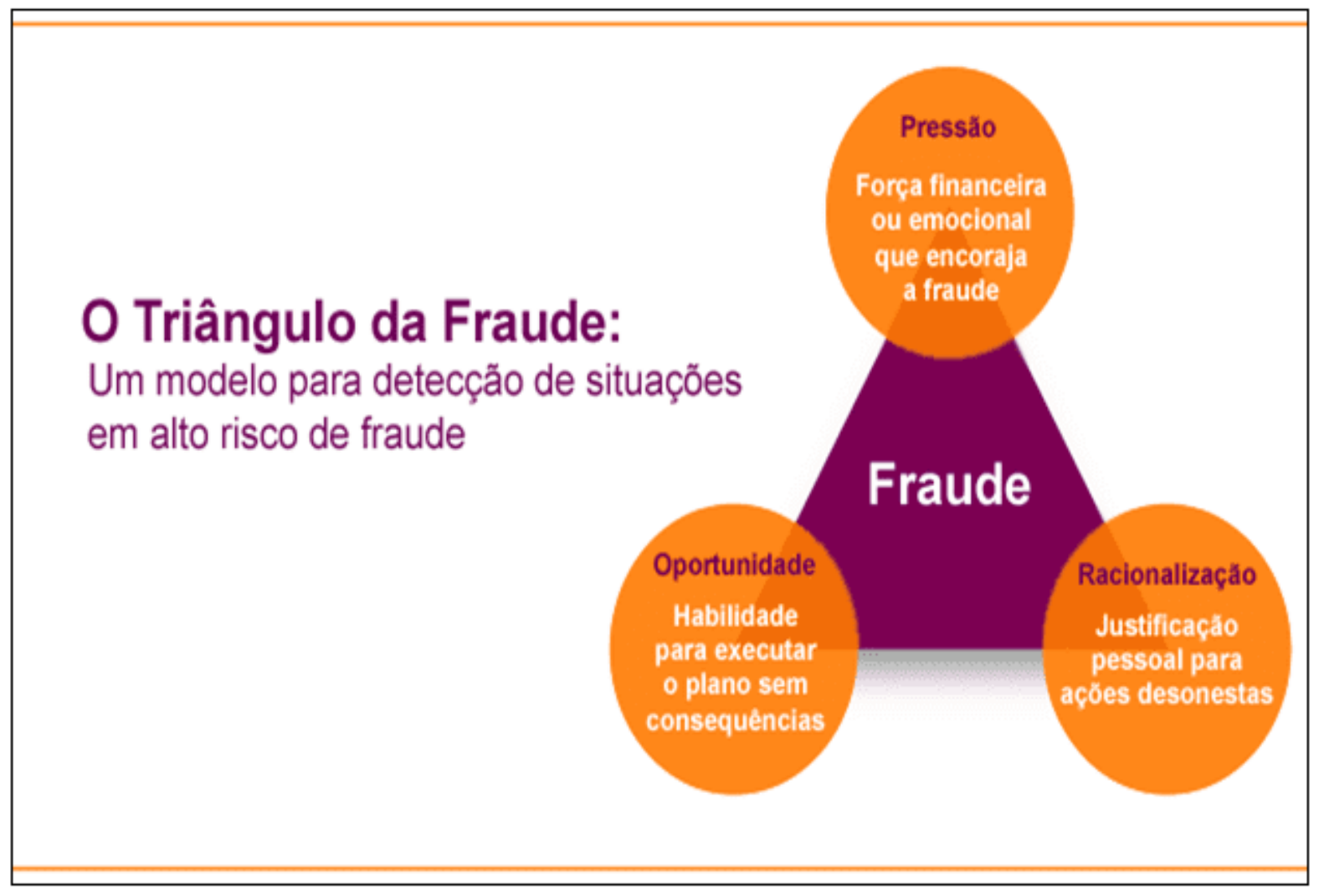

Quelle: Lustosa (2016)

Immer noch Condé et al. (2015) definieren Sie jedes der Elemente, aus denen sich das Betrugsdreieck zusammensetzt:

RC: 103919

Verfügbar in: https://www.nucleodoconhecimento.com.br/business-administrationde/vorteile-von-compliance 
a "pressão" geralmente está ligada ao motivo que leva ao crime, uma necessidade econômica "estressante" da empresa ou do funcionário. A "oportunidade" de cometer o ato ilícito, com baixo risco de ser descoberto ou nenhum. A "Racionalização" de uma autojustificação pelo cometimento do ilícito que para o fraudador seria "aceitável".

In Anbetracht dieses Szenarios stellen wir die Notwendigkeit einer Abbildung organisatorischer Risiken durch die Prävention und Identifizierung von Risiken fest, einschließlich der Nichteinhaltung der Gesetzgebung und des organisatorischen Verhaltenskodex, des operationellen Risikos, des Imagerisikos aufgrund von Unachtsamkeit oder mangelnden angemessenen Verhaltens oder sogar externer Risiken wie der politischen Forderung des Landes. technologische Störungen, Gesundheit und wirtschaftliche Instabilität, Aspekte, die auch Organisationen betreffen können.

In diesem Sinne erklärt Cossenzo (2015), dass zur Verwaltung von Betrug Strategien erforderlich sind, die sie zum gegenwärtigen Zeitpunkt zusätzlich zu anderen identifizieren und ihre Prävention ermöglichen. Wenn die Betrugsrisiken identifiziert werden, ist es auch notwendig zu identifizieren, wie sie auftreten und welche Personen sie ausüben, und dafür ist es wichtig, dass Unternehmen Informationen miteinander teilen.

Derselbe Autor hebt die Existenz der Betrugsbekämpfung Komitees von der Betrug des brasilianischen Bankenverbandes (FEBRABAN), der Basis Kommission für die Inkonsistenz des Nationaler Verband der Kredit-, Finanzierungs- und Investment Institute (ACREFI) und der 2009 zwischen der Bundespolizei und Febraban unterzeichneten Kooperationsvereinbarung in Bezug auf elektronische Kontrollen mit Informationen über die aufgetretenen Fälle hervor (COSSENZO, 2015, S. 35).

In Bezug auf externen Betrug lehrt Oliveira (2012, S. 73-4), auf den sich Cossenzo (2015, S. 36) bezieht, dass es drei präventive Phasen gibt: "Prävention, Erkennung und Reaktion", alle in einer umfassenden Phase, die als "kontinuierlich" bezeichnet wird. Solche Phasen können in Grafik 1 beobachtet werden. 
Tabelle 1 - Phasen und Kategorien der externen Betrugsprävention

\begin{tabular}{|l|l|}
\hline Phase & Verwandte Kategorien \\
\hline Stetig & Verantwortung \\
\hline & Synergie \\
\hline Verhütung & Verbesserung \\
\hline Erkennung & $\begin{array}{l}\text { Institution } \\
\text { Identifizierung }\end{array}$ \\
\hline Reaktion & $\begin{array}{l}\text { Instandhaltung } \\
\text { Forschung }\end{array}$ \\
\hline
\end{tabular}

Quelle: Erstellt vom Autor (2021)

Um solche Vorkommnisse zu verhindern, sollten Maßnahmen ergriffen werden, um die Beteiligten zu sensibilisieren, und es sollten Kontrollen festgelegt und durchgeführt werden, um neue Vorkommnisse zu verhindern. zu ihrer Aufdeckung sollten Ermittlungsmethoden und die Bestätigung des Verdachts auf das Auftreten oder Nichtvorhandensein von Betrug festgelegt werden; und darauf zu reagieren, dass Maßnahmen vom Unternehmen festgelegt werden, wenn Betrug festgestellt wird.

\subsection{LÖSUNGEN FÜR ENTERPRISE GOVERNANCE, RISK MANAGEMENT AND COMPLIANCE (EGRC)}

Zu den Vorteilen, die die Implementierung eines Compliance- und Risikomanagement Programms in Organisationen fördert, gehört der Schutz vor Betrug und illegaler Kriminalität sowie die Förderung der Minderung solcher Risiken.

Damit Sicherheitsmaßnahmen durch eine zunehmend technologische Realität effektiv wirksam sind, ist es notwendig, dass Tools und Lösungen zur Betrugsbekämpfung entwickelt und implementiert werden, die eine bessere Einhaltung der für den Erfolg von Organisationen erforderlichen Praktiken ermöglichen, wie Grand View Research 
(2014) erklärt. Es handelt sich um Maßnahmen, die der InformationstechnologieGovernance sowie dem Internet of things (IOT) oder dem Internet der Dinge innewohnen, die wiederum der Unternehmensführung innewohnen (CHAVES, 2014).

Corporate Governance besteht aus einem "System der Beziehungen zwischen Aktionären, unabhängigen Wirtschaftsprüfern, Führungskräften und Direktoren des Unternehmens unter der Leitung des Verwaltungsrats" (LODI, 2000, bezeichnet von CHAVES, 2014, S. 21). Im Gegenzug beinhaltet IT Governance durch "Big Data und Analysen, loT, maschinelles Lernen sowie KI und soziale Medien den Austausch persönlicher Informationen, wodurch Benutzer und Unternehmen anfälliger für Hacker werden" (GRAND VIEW RESEARCH, 2014).

Die Ressourcen, die das loT und die Digitalisierung von Geschäftsprozessen mit sich bringen, wuchsen schnell, ermöglichten Daten Interaktionen und boten eine bessere Kontrolle der Informationen durch das Management von Unternehmen. In der Praxis besteht das Ziel darin, sich auf Künstliche Intelligenz (KI) zu verlassen, um externe Verpflichtungen und Berichte zu verwalten, die ein besseres Corporate Governance-, Risiko- und Compliance-Management ermöglichen - EGRC (GRANDVIEW RESEARCH, 2014).

Laut Chaves (2014, S.22) "ist IT Governance für das brasilianische Institut für Unternehmensführung (IBGC, 2006) ein Arm der Unternehmensführung", der die Ausarbeitung und Umsetzung von "hochwirksamen Vorschriften im IT-Bereich von Unternehmen ermöglicht, die ein größeres Engagement und eine größere Effektivität bei der Planung, Kontrolle, Überwachung und Sicherheitsprozessen im Zusammenhang mit Geschäftsstrategien erfordern". Immer noch Chaves (2014, S. 22) berichtet über eine Definition des Information Technology Governance Institute (ITGI, 2012) in Bezug auf die Ziele der IT Governance:

compreender os assuntos e a importância estratégica de $\mathrm{TI}$, assegurar que a empresa possa sustentar suas operações e implementar as estratégias requeridas para expandir as suas atividades no futuro. As práticas de Governança de TI possibilitam que: sejam atendidas as expectativas dos

RC: 103919

Verfügbar in: https://www.nucleodoconhecimento.com.br/business-administrationde/vorteile-von-compliance 
clientes de TI, que a performance de TI seja medida, os seus recursos possam ser gerenciáveis e os riscos do negócio mitigados.

Seinerseits. Siqueira (2019) bezieht sich auf die Daten- und Cyber Sicherheitsrisiko Umfrage, die 2018 von Marsh und McLennan erstellt wurde und zeigt, dass nur 18\% der kleinen Unternehmen einen Reaktionsplan für Cybervorfälle entwickelt hätten.

In dieser Hinsicht bietet die Erstellung und Implementierung von EGRC-Lösungen die Möglichkeit, bestehende Herausforderungen - wie beispielsweise elektronischen Betrug und Klonen - in einem "hyper verbunden" Geschäfts Universum zu überwinden, das durch spezifische Software bezogen werden kann. Es stellt auch ein Instrument dar, das wesentlich im Bereich Datenschutz und geistiges Eigentum (GE) zusammenarbeitet, zusätzlich zur Verbesserung des Managements von RemoteArbeit, einem hybriden Modell, das während der Pandemie durch Covid-19 so verbreitet war (GRAND VIEW RESEARCH, 2014).

\section{VORTEILE EINES COMPLIANCE- UND RISIKOMANAGEMENT PROGRAMMS}

Laut dem Portal 3Mind Legal (2021) gehört zu den Hauptkonzepten von Compliance und Risikomanagement die Generierung von Geschäftswert, der darauf abzielt, das Überleben und die Langlebigkeit von Organisationen zu ermöglichen. Zu den Gefahren und finanziellen Auswirkungen, die das Fehlen eines Programms zur Förderung guter Regierungsführung hat, gehören: "Fehlen normativer Richtlinien; Mangel an angemessenen Präventionsinstrumenten; Fehlausrichtungen mit den geltenden Gesetzen; Informationssystem ohne strukturierten Betrieb; Management fehlgeschlagener Prozesse."

Da öffentliche und private Institutionen ihr Management in Corporate-GovernanceProgrammen leiten sollten, Klen (2009, apud SENNO et al., 2019, S. 225) definiert, dass "Corporate Governance das System ist, nach dem Unternehmen geführt und kontrolliert werden". 
In diesem Sinne sind immer noch Senno et al. (2019) erläutern, dass die CorporateGovernance-Praktiken konvergieren müssen, um Mechanismen zu gewährleisten, die die finanzielle Rendite der getätigten Investitionen durch die Annahme von Rechtsvorschriften und die Rechenschaftspflicht gegenüber allen Interessenträgern ermöglichen.

Azevedo et al. (2017, S. 181) erklären, dass sich Governance auf die Regierung bezieht, während sich Corporate Governance auf das System bezieht, nach dem Organe und Befugnisse innerhalb eines Unternehmens organisiert sind", das durch vier Prinzipien gestützt werden muss: Transparenz, Integrität oder Gerechtigkeit, Rechenschaftspflicht und Einhaltung von Gesetzen.

Diese Erklärungen zielen darauf ab, zusammenzuarbeiten, um good governance Practices besser zu verstehen, die laut KPMG (2021) einige spezifische Elemente erfordern, um ein effizientes Regulatory Compliance-Programm zu bilden, nämlich:

1. Compliance-Richtlinie und -Programm;

2. Botschaft der Führung und Kultur der Compliance;

3. Strukturierung des Compliance Officers;

4. Kommunikation und Schulung der Compliance-Richtlinie und des ComplianceProgramms;

5. Überwachung, Prüfung und Bewertung der Wirksamkeit der Ethik- und Compliance-Richtlinie und des Programms;

6. Anreize für Leistungs- und Disziplinarmaßnahmen;

7. Forschung und Prävention.

In dieser Hinsicht wird berichtet, dass ein Compliance-Programm Anti-Korruptions-, Anti-Bestechungs- und öffentliche Compliance-Standards impliziert. Für weitere Details sind die von Compliance bereitgestellten Vorteile laut dem Portal 3Mind Rechtliches (2021) aufgeführt:

1. Investoren und Investitionen anziehen;

2. Erhöhung der Governance im Unternehmen;

RC: 103919

Verfügbar in: https://www.nucleodoconhecimento.com.br/business-administrationde/vorteile-von-compliance 
3. Konsolidierung der Unternehmenskultur;

4. Effektive Korrektur von Nichtkonformitäten;

5. Glaubwürdigkeit gewinnen;

6. Verschaffen Sie sich einen Wettbewerbsvorteil gegenüber der Konkurrenz;

7. Risiken identifizieren und Problemen vorbeugen;

8. Verbesserung der Effizienz und Qualität der angebotenen Produkte und Dienstleistungen;

9. Nachhaltigkeit.

Im Gegenzug beschreibt Caovilla (2017) einige Vorteile im Zusammenhang mit der Einführung von Compliance durch Organisationen (unsere Greifen):

1. Neue Geschäftsmöglichkeiten und Wettbewerbsvorteile: Unternehmen, die in Übereinstimmung mit Gesetzen und bewährten Verfahren arbeiten, versuchen, sich auf gleichberechtigte Paare zu beziehen und ihre Chancen gegenüber denjenigen zu erhöhen, die keine Compliance-Programme nutzen.

2. Neue Investitionen: Organisationen mit geringen Beteiligungsraten an illegalen und sozialen Skandalen, die Solidität demonstrieren, sind für interne und externe Investoren attraktiver;

3. Risikominderung: Unternehmen, die ein Compliance-Programm einführen, zusätzlich zur Identifizierung möglicher Risiken für ihr Geschäft in der Lage sind, Maßnahmen zur Minderung von Fehlern und Risiken zu entwickeln und umzusetzen;

4. Korrektur von Nichtkonformitäten: Neben vorbeugenden Maßnahmen zur Vermeidung möglicher Nichtkonformitäten sind die Maßnahmen zur Korrektur der in den internen Prozessen einer Organisation identifizierten Nichtkonformitäten enthalten. Solche Maßnahmen, die notwendig sind, um das Image und den Ruf eines Unternehmens zu erhalten, können sein: Korrektur des laufenden Aktionsplans; Schulung der Mitarbeiter; Überprüfung von Richtlinien, Verfahren, Verhalten, Mitarbeitern und Lieferanten und Dienstleistern; 
5. Compliance als Bewusstsein der Menschen: eine Praxis, die Betrugsbekämpfung ermöglicht, da Mitarbeiter und andere Akteure, wenn sie in Compliance geschult sind, in der Lage sind,Nichtkonformitäten innerhalb und außerhalb des Unternehmens zu sehen, dh im Wettbewerb, bei Lieferanten, Dienstleistern;

6. Compliance als Haftungsbeschränkung: Kenntnisse, die in Schulungen und ethischen Praktiken im Zusammenhang mit Compliance erworben wurden, ermöglichen die Auswahl besserer Geschäftspartner und schützen den Ruf eines Unternehmens. Da gute Praktiken die Geschäftsabläufe einer Organisation durchdringen, kann jede Nichtkonformität zur Verringerung der Strafen führen, die auf sie angewendet werden können.

7. Compliance als unternehmerische Nachhaltigkeit: Die Einführung und Aufrechterhaltung ethischer und transparenter Praktiken ermöglicht es Unternehmen, langfristig zu bleiben.

\section{FAZIT}

Während der Analyse der theoretischen Referenzen, die für die Entwicklung dieses Artikels ausgewählt wurden, konnte festgestellt werden, dass ein Compliance- und Risikomanagement Programm eine hohe Bedeutung für das ordnungsgemäße Management von Prozessen, Praktiken und Routinen innerhalb von Organisationen unter Einbeziehung aller ihrer Sektoren und Mitarbeiter hat.

Rückkehr zum Punkt der Zügel; Wie wirken die Vorteile des Compliance- und Risikomanagement Programms zusammen, um die Risiken von Betrug, illegaler Kriminalität und Korruption in Regierungsorganisationen und -institutionen zu reduzieren? Wir kommen zu dem Schluss, dass, da die Literatur zeigt, dass es in allen Organisationen Betrügereien gibt, ohne Unterschied für das Tätigkeitssegment oder seine Größe, der Vorteil, den Compliance den Unternehmen bietet, genau in dem Schutz liegt, den es bietet, indem die verschiedenen Arten von Risiken und illegalen Risiken gemindert werden, einschließlich der Vermeidung rechtlicher Sanktionen.

RC: 103919

Verfügbar in: https://www.nucleodoconhecimento.com.br/business-administrationde/vorteile-von-compliance 
Auf der Grundlage der in diesem Artikel aufgeführten Gesetze, aus dem Pionierrecht zur Bekämpfung der Korruption FCPA (Foreign Corrupt Practices Act), haben die in diesem Artikel aufgeführten Unterzeichnerstaaten der OECD ähnliche Initiativen ergriffen, um die Eindämmung von Betrug, Korruption und unethischen Handlungen in ihren Regierungen und Organisationen zu fördern, die durch die Einschränkungen und Strafen der Gesetze stark unterstützt werden.

Darüber hinaus entstand die Entwicklung neuer technologischer Instrumente, unterstützt durch Information Technology Governance, um die Corporate Governance zu unterstützen und Veränderungen im Management von Organisationen und ihren Verwaltungsprozessen zu fördern.

Ein solches Management ist etwas, das auf modernen Tools basieren sollte, die nicht auf die Prozesse selbst beschränkt sind, sondern es einer Organisation ermöglichen sollten, zukünftige Ereignisse zu antizipieren und Missverständnisse zu vermeiden.

In diesem Zusammenhang arbeiten EGRC-Lösungen- und Best Practices für ITGovernance und Corporate Governance zusammen, um Betrug und unvorsichtige Maßnahmen zu verhindern und zu mildern und das Wachstum von Organisationen und ihr Marktimage zu schützen.

\section{VERWEISE}

AZEVEDO, Mateus Miranda de; CARDOSO, Antonio Almeida; DUARTE, Jairo Gonçalves; FEDERICO, Bianca Ellen; LIMA, Marco Antonio Ferreira. O Compliance e a gestão de riscos nos processos organizacionais. Revista de Pós-Graduação Multidisciplinar, São Paulo, v. 1, n. 1, p. 179-196, mar./jun. 2017.

ISSN 2594-4800 | e-ISSN 2594-4797 | doi: 10.22287/rpgm.v1i1.507; acesso em 08 dez 2021.

RC: 103919

Verfügbar in: https://www.nucleodoconhecimento.com.br/business-administrationde/vorteile-von-compliance 
CAOVILLA, Renato Vieira. Benefícios práticos do compliance. 10 jul 2017. Disponível em: [https://www.migalhas.com.br/depeso/261662/beneficios-praticos-docompliance]; 08 dez 2017.

CHAVES, Elisabete Cecília Januário. Resultados e tendências de Requisitos e Práticas de Governança de TI nos bancos de Varejo no Brasil. Dissertação [Mestrado em Gestão e Tecnologia em Sistemas Produtivos] apresentada ao Centro Estadual de Educação Tecnológica Paula Souza, no Programa de Mestrado Profissional em Gestão e Tecnologia em Sistemas Produtivos. São Paulo, novembro de $2014 . \quad$ Dsponível em: [http://www.pos.cps.sp.gov.br/files/dissertacoes/file/143/7fc218f434af24b99f50af42e 8442903.pdf]; acesso em 12 out 2021.

COELHO, Cláudio Carneiro Bezerra Pinto; SANTOS JÚNIOR, Milton de Castro. Compliance, FGV/IDE, 2021.

CONDÉ, Robson Augusto Dainez; ALMEIDA, Carlos Otávio Ferreira de; QUINTAL, Renato Santiago. Fraude Contábil: análise empírica à luz dos pressupostos teóricos do triângulo da fraude e dos escândalos corporativos. Gestão \& Regionalidade - Vol. 31 - № 93 - set-dez/2015. doi: 10.13037/gr. vol31n93.2929; acesso em 28 out 2021

COSSENZO, Fábio. FRAUDE BANCÁRIA: A mitigação dos Riscos e Perdas Financeiras no Segmento de Crédito Consignado. Monografia de Pós-Graduação Lato Sensu - MBA Gestão empresarial, apresentada à Fundação Instituto de Administração - FIA. São Paulo, 2015.

DURÃES, Cintya Nishimura; RIBEIRO, Maria de Fátima. O Compliance no Brasil e a Responsabilidade Empresarial no combate à corrupção. Revista Direito em Debate: Revista do Departamento de Ciências Jurídicas e Sociais da Unijuí Editora Unijuí - Ano XXIX - n. 53 - jan./jun. 2020. Disponível em: [http://dx.doi.org/10.21527/2176-6622.2020.53.69-78]; acesso em 01 dez 2021. 
ENTERPRISE GOVERNANCE, RISK \& COMPLIANCE MARKET SIZE. Share \& Trends Analysis Report By Component, By Software, By Services, By Enterprise Type, By Vertical, And Segment Forecasts, 2021 - 2028

FGV - FUNDAÇÃO GETÚLIO VARGAS. Surgimento do Compliance. 2021.

GONSALES, Alessandra. O que é compliance? Publicação da Legal, Ethics and Complaince (LEC). Vídeo publicado em 29 fev 2016. Disponível em: [https://www.youtube.com/watch?v=2BDpJ6UMXb4]; acesso em 01 dez 2021.

GRAND VIEW RESEARCH. Relatório de governança corporativa, risco e conformidade do mercado, análise de compartilhamento e tendências por componente, por software, por serviços, por tipo de empresa, por vertical e por segmento. Previsões, 2021-2028. Artigo publicado em abril de 2021. Disponível em: [https://www.grandviewresearch.com/industry-analysis/enterprise-governance-riskcompliance-egrc-market]; acesso em 20 nov 2021.

HAUSSEN, Leonard. Compliance. Reunião On-Line (ROL), Aula Organizada pela Fundação Getúlio Vargas, Escola de Administração de Empresas de São Paulo, São Paulo, Brasil. Agosto de 2021.

JORGE, Glaucio Fiorenzano; TOMAZ, Roberto Epifânio. Compliance rins - como implantar e quais os benefícios do programa de compliance? IV Congresso Catarinense de Direito processual Civil \& Mais. 7 a 9 de novembro de 2018. Univali Eventos - Universidade Vale do Itajaí. Disponível em: [https://core.ac.uk/download/pdf/276005736.pdf]; acesso em 20 out 2021.

KPMG - Compliance analytics. 2018. Disponível em: [https://assets.kpmg/content/dam/kpmg/br/pdf/2018/08/br-compliance-analytics.pdf]; acesso em 08 dez 2021. 
KPMG - Compliance regulatório. 2021. Disponível em: [https://home.kpmg/br/pt/home/servicos/consultoria/risk-consulting/internal-auditrisk/compliance-regulatory.html]; acesso em 08 dez 2021.

MELO, Luísa. 8 crises empresariais causadas por funcionários brincalhões. Matéria publicada em 14 mar 2014. Disponível em: [https://exame.com/negocios/8crises-empresariais-causadas-por-funcionarios-brincalhoes/]; acesso em 20 out 2021.

MICHELOTTI, Andriws Loreto. 0 crime de lavagem de capitais e os desafios empresariais diante da implementação dos programas de criminal Compliance. Trabalho de Conclusão de Curso [Bacharelado em Direito] apresentado à Faculdade Antonio Meneghetti (AMF). Restinga Seca (RS), 2020. Disponível em: [http://repositorio.faculdadeam.edu.br/xmlui/bitstream/handle/123456789/673/TCC_D IR_ANDRIWS_MICHELOTTI_AMF_2020.pdf?sequence=1\&isAllowed=y]; acesso em 07 dez 2021.

NASCIMENTO, Juliana Oliveira. A Função Sociald da Empresa e a Efetividade Prática da Conduta de Integridade: o Compliance Vivo. Dissertação [mestrado em Direitos Fundamentais e Democracia] apresentada ao Centro Universitário Autônomo do Brasil. Curitiba, 2018. Disponível em: [https://www.unibrasil.com.br/wpcontent/uploads/2019/07/Dissertação-JULIANA-OLIVEIRA-NASCIMENTO.pdf]; acesso em 29 ago 2021.

NASCIMENTO, Débora Minuncio. Evolução histórica e legislações acerca do Compliance. Artigo publicado em 07 nov 2021. Disponível em: [https://advocaciadeboramn.jusbrasil.com.br/artigos/700763578/evolucao-historica-elegislacoes-acerca-do-compliance]; acesso em 20 out 2021.

\section{OECE - ORGANIZAÇÃO PARA A COOPERAÇÃO E DESENVOLVIMENTO ECONÔMICO INTERNACIONAL. \\ Disponível \\ em:}

[https://www.dgae.gov.pt/servicos/comercio-internacional-e-relacoesinternacionais/multilaterais/organizacao-para-a-cooperacao-e-desenvolvimentoeconomico-ocde-.aspx]; acesso em 08 dez 2021.

RC: 103919

Verfügbar in: https://www.nucleodoconhecimento.com.br/business-administrationde/vorteile-von-compliance 
PwC. Combate a fraudes e crimes econômicos: uma batalha sem fim Pesquisa Global sobre Fraudes e Crimes Econômicos 2020. Disponível em: [https://www.pwc.com.br/pt/publicacoes/servicos/assets/consultorianegocios/2020/pesquisa_sobre-fraudes-e-crimes-economicos-2020-pwc-brasil.pdf]; acesso em 20 out 2021.

SENNO, Elisângela Pereira; PRATES, Glaucia Aparecida; LUCENTE, Adriano dos Reis; GALLI, Lesley Carina do Lago Attadia; GALLI, Rafael Altafin. Benefícios da implantação do programa de Compliance em uma organização do setor de energia: um estudo de caso. II SITEFA - Simpósio de Tecnologia Fatec Sert5aozinho. Disponível em: [https://sitefa.fatecsertaozinho.edu.br/index.php/sitefa/article/view/90/51]; acesso em 08 dez 2021.

SIQUEIRA, Marina Paiva de. Cyber Risks: Fatores decisórios para a transferência mediante a contratação do seguro. Monografia [Bacharelado em Administração] apresentada ao Departamento de Administração da Universidade Federal Fluminense. Niterói, RJ. 2019.

SOBREIRA FILHO, Enoque Feitosa; LEITE, Flavia Piva Almeida; MARTINS, José Alberto Monteiro. Ética empresarial como base de sustentação do programa de Compliance: uma breve análise sobre a ética, a integridade e o Compliance. Revista Relações Internacionais no Mundo Atual. Vol. 2,n. 23, 2019. Disponível em: [http://revista.unicuritiba.edu.br/index.php/RIMA/article/view/3891/371373033]; acesso em 29 ago 2021.

SOUZA, Silvia Regina; MACIEL-LIMA, Sandra; LUPI, André Lipp Pinto Basto. Aplicabilidade do Compliance na Administração Pública em face ao momento político atual brasileiro. Percurso - Anais do I Congresso Ibro-Americano de Direito Empresarial e Cidadania (CONIBADEC), vol. 01, n. 24, Curitiba, p.1-22, 2018. Disponível em: 
[http://revista.unicuritiba.edu.br/index.php/percurso/article/view/3274/371371782]; acesso em 01 dez 2021.

3MIND JURÍDICO. Compliance: o que é e o benefício para empresas. Abril de 2021. Disponível em: [https://www.3mind.com.br/blog/compliance-empresas/]; acesso em 08 dez 2021.

VAZ, Tatiana. 5 empresas envolvidas em escândalos recentes. Artigo publicado em 30 abr 2012. Disponível em: [https://exame.com/negocios/5-empresas-envolvidasem-escandalos-recentes/]; acesso em 28 out 2021.

VEJA ON LINE. Funcionário de restaurante publica foto lambendo comida de clientes. Junho de $2013 . \quad$ Disponível em: [https://veja.abril.com.br/economia/funcionario-de-restaurante-publica-fotolambendo-comida-de-clientes/]; acesso em 14 out 2021.

Eingereicht: November 2021.

Genehmigt: Dezember 2021. 